\title{
SOME RECENT ADVANCES IN PHYSICAL SCIENCE.*
}

\author{
By Frederick H. Getman, Ph.D. \\ Stamford High School, Stamford, Conn.
}

Many and various have been the discoveries made in physical science during the last decade and any attempt to review them ever so briefly would consume much more time than is at our disposal. Although physicists have been engaged with research in all of the different branches of physics we find the greatest amount of attention being directed to the study of the passage of electricity through gases and to the allied and equally fascinating phenomena of radioactivity.

To some of these researches I ask your attention, and for my failure to mention much excellent work along these lines I must make lack of time my excuse.

\section{ROENTGEN RAYS.}

While the study of the passage of electricity through gases at low pressures dates back to the early work of Harris in 1834 and of Faraday in 1838 it was not until Roentgen's epochmaking discovery of the X-ray in 1895 that high vacua discharge became the subject of almost universal study.

The observation that photographic plates became fogged in the neighborhood of a highly exhausted tube through which electric discharges were passing led Roentgen to investigate the type of radiation which seemed to proceed from the tube. $\mathrm{He}$ found that the bombardment of the walls of the tube by the cathode rays produced a form of radiation capable of penetrating: many substances which are opaque to light. Aside from their penetrating power the Roentgen or X-rays produce luminescence in barium platino-cyanide and several other salts, this property being made use of in the well known fluoroscope. It was also discovered that the X-ray is capable of discharging an electrified body on an insulated support. The physical nature of the $\mathrm{X}$-ray has been the subject of much experimental work. Since the rays are neither reflected nor refracted and since it is only with great difficulty that they have been made to exhibit slight signs of polarization we would be inconsistent were we to identify them with ordinary light. On the other hand we are not

\footnotetext{
* Paper read before the Physics section of the Connecticut Association of Classical and High School Teachers. New Haven. Feb. 16. 1907.
} 
justified in considering them as a flight of material particles since they are not deviated by a magnetic or an electric field.

In 1896 Sir George Stokes suggested that the X-rays should be looked upon as single, irregular ether pulses propagated with the speed of light.

J. J. Thomson has shown that by the sudden stoppage of a moving electrified particle, an expanding spherical shell of electromotive force would start and radiate from the particle with the velocity of light. Within the shell electric and magnetic forces exist at right angles to each other and also to the direction of propagation. Stokes has shown that such disturbances would have many of the properties possessed by the $\mathrm{X}$-ray.

According to this theory the character of the ether pulse should be dependent upon the character of the arrest of the electrified particle. In support of this requirement of the theory may be cited the well known fact that the character of the radiation is dependent upon the degree of exhaustion of the tube, the so-called "hard rays" being obtained with high exhaustions.

\section{CATHODE RAYS.}

Crookes advanced the idea that the cathode rays consist of a stream of electrified particles and the discovery of Roentgen, together with the conceptions of Stokes as to the physical characteristics of the X-ray, only confirmed this suggestion. It was the natural consequence of Roentgen's discovery that the cathode rays should receive closer attention than ever before and in the course of experimentation M. Perrin gave a direct proof that the cathode rays carry a negative charge. $\mathrm{He}$ showed that when the rays within the tube were deflected by a magnet so as to fall within a metallic screen connected with the earth, an instulated metallic cylinder inside the screen exhibited a strong negative electrification and when the rays fell on other parts of the tube the electrometer showed no sign of electrification.

We now turn our attention to the experiments carried out in the Cavendish laboratory of Cambridge University under the skillful direction of $\mathrm{J}$. J. Thomson. He repeated the experiment of Perrin subjecting the cathode rays successively to the action of a magnetic and an electric field. From the results of 
this experiment it is possible to calculate the velocity of the cathode particles, to determine the nature of their charge and to measure the ratio of $e$, the charge carried, to $m$, the mass of the carrier. The results obtained from this experiment are so important that it seems advisable to outline the method as briefly as possible.

Imagine a single electrified particle to be moving in a rectilinear path with uniform speed $v$. Let us now apply an electric field of uniform intensity, $f$, at right angles to the direction in which the particle is moving. The force on the particle is $f e$ and the acceleration, $a$, at right angles to the initial path is given by $\underset{m}{f e}$ or the particle traces a parabolic orbit. The electric displacement is then $d_{\mathrm{e}}=\frac{1}{2} a t^{2}=\frac{1}{2} \frac{f e}{m} t^{2}$.

In the interval of time $t$ the particle traverses a distance $l=v t$; from which we get $t^{2}=\frac{l^{2}}{v^{2}}$ or $d_{\theta}=\frac{1}{2} \frac{f e}{m} \frac{l^{2}}{\eta^{2}} \ldots$ (1).

Equation (I) involves, the electric intensity, the velocity of the particle and the ratio of charge to mass.

We now replace the electric field by the magnetic field of intensity $H$, also at right angles to the path of the particle. The particle moving with charge $e$ at speed $v$ is equivalent to a current $C$ passing over a path $d l$ or $e v=c d l$.

In this case the force of the particle is $H e v$ and is at right angles to both the magnetic force and the direction of motion. The direction constantly changes similar to the pull on a string by which a stone is whirled. Therefore the expression for centrifugal force, $\frac{m v^{2}}{r}$ applies and we have $H e v=\frac{m v^{2}}{r}$ or $r=\frac{m v}{e H}$.

The magnetic deflection is $d_{\mathrm{m}}=\frac{l^{2}}{2 r}=\frac{l^{2} H}{2 \tilde{y}} \cdot \frac{e}{m} \ldots$. (2).

Here we have a new relation between $v$ and $\frac{e}{m}$ thus enabling us to calculate both $v$ and $\frac{e}{m}$ in absolute units.

In carrying out the experiment Prof. Thomson applied both electric and magnetic fields simultaneously, so adjusting the lat- 
ter that it exactly balanced the former and no deflection took place.

Under these conditions $f e=H e v$ or $v=\frac{f}{H}$ and $\frac{e}{m}=\frac{2 v d_{m}}{l^{2} H}$.

The results of these experiments show that $v$ may vary con. siderably, from $2 \times 10^{\circ}$ to $4 \times 10^{\circ}$ centimeters per second, while the quantity of $\frac{e}{m}$ remains constant. 'It has been shown that the ratio $\frac{e}{m}$ is independent of the nature of the gas and of the materials of which the cathode is made, the value of the ratio being about $7.7 \times 10^{8}$.

The passage of one electro-magnetic unit of electricity in electrolysis liberates approximately $10^{-4}$ grams of hydrogen and the value of $\frac{e}{m}$ is therefore $10^{4}$.

From this we see that the value of $\mathrm{e} m$ for the cathode rays is nearly 800 times greater than the corresponding value for the hydrogen ion in electrolysis.

Were it possible to determine the charge, carried by the cathode particle it is evident that we could determine its mass. This problem has been successfully solved by Thomson, the method employed and the results obtained being outlined later.

\section{IONIZATION OF GASES.}

Under ordinary circumstances air and other gases are very poor conductors. If due precautions are taken to prevent leakage along the supports electrified bodies maintain their charges over considerable periods of time.

C. T. R. Wilson in the construction of the electroscope bearing his name has made use of the remarkable insulating properties of sulphur, and has developed an instrument in which all tendency for leakage along the supports has been removed.

But even here we note a slow but continuous loss of charge which can only be explained on the assumption that air possesses slight conductivity. The hot gases arising from flames have long been known to discharge electrified bodies and attention has already been recalled to the action of Roentgen rays upon a charged electroscope. Ultra-violet light has been found to exert a similar action upon charged bodies and the recently discovered radio-active substances are remarkably efficient in this respect. 
It has been shown that gases which have been subjected to the action of any of these agencies do not part immediately with their power of discharging the electroscope but may be blown considerable distances through tubes and still be capable of causing the leaves of the electroscope to collapse. From these and similar experiments we infer that gases undergo a process of ionization resembling that experienced by electrolytes.

It is possible, however, to remove the gaseous ions by contact with solids or liquids or through the action of an electric field. There is also a gradual disappearance of ionization without any of the agencies just mentioned. This last fact has led to the hypothesis that the positive and negative ions recombine under the attractive forces of opposite charges. The conception has been subjected to mathematical treatment and from the experiments of Prof. Rutherford the hypothesis has been confirmed, it having been shown that if the concentration be $10^{\circ}$ ions per cubic centimeter, half of them recombine in one second.

\section{Conductivity of Gases.}

In accordance with Ohm's law the current passing through a gas should be directly proportional to the electro-motive force applied. This point was tested by Thomson and Rutherford by ionizing the air between two parallel metal plates by means of the X-rays. One plate was connected with one pair of quadrants of an electrometer, the other pair being connected with the earth, the second plate was connected with one pole of a battery, the remaining pole being earthed.

With this arrangement of apparatus it was found that for small potential-differences the gas obeys Ohm's law but as the potential-difference increases the current increases more and more slowly. Finally the current becomes constant until the difference in potential is great enough to cause a spark to pass, when it increases at a rate greater than that demanded by Ohm's law. When the current attains to saturation it becomes independent of the intensity of the voltage, a fact which renders the determination of the degree of ionization of a gas a matter of considerable ease. All that is necessary is to assure oneself that the saturation value has been slightly exceeded and then the current can be measured either by the rate of deflection of the eiectrometer needle or by the rate of collapse of the gold leaves 
of an electroscope. This method has been adopted by the Curies and their colleagues in determining the activity of various radioactive preparations. It has been found that the ionization current increases with increase in distance between the plates, a result which is in complete accord with the theory, since the increase in space affords more room for the ionizing agent and thus augments the number of ions.

From the experiments of Rutherford and Zeleny it is possible to calculate the velocities of the individual ions the results obtained by the two workers being in very good agreement. It has been shown that for pressures greater than a small fraction of an atmosphere the speed of an ion varies inversely as the pressure of the gas through which it moves. This statement when compared with the well known fact that the internal friction of a gas is independent of the pressure shows that there is no analogy between the motion of the gaseous ion and the gaseous molecule.

It has been shown that the impact of the cathode particles upon the molecules of a gas is sufficient to cause ionization. If two metal plates be separated by a layer of gas and a difference of potential be-set up between them the few ions which are present in the gas are set in motion, the positive ions moving to the negative plate and the negative ions to the positive plate. As the pressure is diminished the longer the free path of an ion becomes before colliding with a molecule of the gas and hence the greater its velocity. When collision does take place the greater the impact and the greater will be the ionization.

If the pressure be sufficiently reduced the result will be that the supply of ions will continually increase and eventually a continuous electric discharge will pass through the exhausted gas. The potential-gradient required to produce ions by collision at atmospheric presure amounts to approximately 30,000 volts per centimeter.

\section{Condensation Nuclei.}

Some years ago Aitken observed that dust particles in the air aided the formation of fog. If no dust were present a condition of supersaturation could be reached if the air were subjected to sudden adiabatic expansion.

These early observations of Aitken were extended by C. T. R. Wilson, who pointed out that if the air were rendered dust-free 
by preliminary expansion an increase of volume from I to 1.38 was necessary to cause the formation of a dense fog or cloud. On the other hand, if the air were ionized the cloud. formed at an expansion of 1.25. In other words, the gaseous ions can replace the dust particles as condensation nuclei. Some years ago Sir George Stokes investigated mathematically the friction of a sphere moving through a viscous liquid. He pointed out that as a spherical drop falls its velocity increases and at the same time the friction increases until finally a steady state is reached when the force due to the weight of the drop is equal to the friction. Under these conditions the velocity of the drop is uniform and by observing the rate of fall of the drop we can calculate its radius.

Wilson applied this method to the determination of the number of nuclei or ions in the clouds formed in his fog chamber. From the value of the expansion used and the total volume of the fog chamber it is easy to calculate the volume of the water condensed. Dividing the total volume by the volume of each drop we obtain the number of drops and as each ion acts as the nucleus of a drop we know the number of ions. This method was employed by Thomson in determining the number of ions per cubic centimeter of a gas. Knowing their velocity under definite potential and measuring the current through the gas under known potential he was able to calculate $e$, the ionic charge.

The value obtained for $e$ agrees closely with the corresponding value of $e$ for the hydrogen ion in electrolysis.

We have already seen that the value of $\frac{e}{m}$ for the gaseous ion and for the hydrogen ion in electrolysis are to each other as $800:$ I and since the charge is the same in each case we are led to the very important conclusion that the gaseous ion is nearly one one-thousandth of the mass of the hydrogen atom.

\section{CORPUSCLES.}

Thomson has confirmed his results by studying the ionization produced by ultra-violet light falling on a metal plate in a bighly exhausted vessel. By this method he was able to measure $e$ and $\frac{e}{m}$ for the same particle. The agreement between the two methods is surprising, the value of $\frac{e}{m}$ by the first 
method being $7.7 \times 10^{6}$ and by the method just mentioned $7.3 \times$ $10^{\circ}$.

We are thus forced to the conclusion that the chemical atom is made up of from eight hundred to a thousand smaller particles which Thomson calls corpuscles. Associated with these corpuscles is a negative electric charge which appears to play the part of a true unit of electricity. Although search has been made for it, the corresponding positive corpuscle has never been tound, the positive ion thus seeming to possess atomic dimensions.

These facts have been explained on the assumption that the corpuscle represents a unit negative charge of electricity.

Thus an atom with one corpuscle in excess of its normal number possesses a negative charge, while an atom which is deficient by one corpuscle is positively charged.

Since experiments have shown that the mass of the corpuscle is independent of the nature of the gas or of the electrode used in producing, it we are led to the further conclusion that all matter is made up of similar ultimate particles.

This belief in the ultimate particle has existed for years largely through man's scientific intuition but today Thomson furnishes us with undeniable evidence of the truth of our longcherished conception. In I887 Sir William Crookes wrote: "I venture provisionally to conclude that our so-called elements or simple bodies are, in reality, compound molecules. What existed anterior to our elements, before matter as we now have it, I propose to name Protyle." Thomson's corpuscle seems to fill the requirements of the protyle of Crookes.

The corpuscular theory may be employed to explain why it is that the absorption of cathode rays is proportional to the density of the absorbing substance, while no such relation exists for light. We know that gold, silver, iron and glass, all of them heavy bodies, or paper, cork or water, all light substances, may be either opaque or transparent to light. On the other hand, these substances absorb cathode rays or the radiation from radioactive bodies in direct proportion to their densities.

Until the evolution of the corpuscular theory it was difficult to see how these facts could be explained. Under the present conception of the atom, it is to be looked upon as a miniature solar system in which the corpuscles play the part of satellites to a central nucleus. The inter-corpuscular spaces within the atom 
are thus very great in comparison with the dimensions of a single corpuscle so that when the cathode particles bombard a sheet of metal they find their way through the atomic structure.

The absorbing powers must thus be proportional to the density of the absorbing material and also independent of its other properties.

\section{IONIZATION CAUSED BY INCANDESCENCE.}

It has been shown that the hot gases from a flame and the air near an incandescent solid will discharge an electrified body. Eister and Geitel have studied the effect produced by a platinum wire as it is gradually heated. At a low red heat it gives off positive ions and then as the wire is heated further, negative ions begin to be emitted until at high temperatures the latter predominate. It has been found that in a vacuum the negative leakage from platinum filaments is very large, amounting in some cases to as much as an ampere per square centimeter. These negative ions are to be looked upon as identical with corpuscles.

Not only do solids emit corpuscles at high temperature but also gases and vapors are capable of giving off a large supply. Arrhenius has pointed out that this class of phenomena has a very important bearing on cosmic processes.

The photosphere of the sun contains carbon heated to an extremely high temperature and thus is capable of emitting corpuscles. This emission of negative electricity will go on until the electrostatic attraction of the residual positive charge is sufficient to bring about a condition of equilibrium.

Any sudden change in temperature would obviously disturb this equilibrium, corpuscles passing either outward or inward as the temperature rises or falls. It is known that when corpuscles pass through the gas with high speed they render it luminous. Arrhenius explains the Aurora Borealis on the supposition that corpuscles from the sun stream through the upper regions of the atmosphere and cause it to become luminous.

METALLIC CONDUCTION.

The phenomena of electrolytic conduction must now be interpreted in terms of the new corpuscular theory.

The cation must consist of an atom of the electrolyte minus one of its corpuscles while the anion must be regarded as an atom of the electrolyte plus its associated corpuscle. 
The cation is positive because it has lost one unit of negative electricity. The modern theory of electricity brings to mind the old "single fluid" theory in which the terms positive and negative were used to designate an excess or a deficiency of electricity. In fact it would only be necessary to revise the meaning of the terms positive and negative to make the old conventions fit the new theory.

It has been difficult to explain metallic conduction by means of the phenomena of electrolytic conduction in liquids but in the light of the new corpuscular theory we may form a fairiy clear conception of the process.

We imagine the corpuscles to be freely mobile within the substance of the conductor and when a difference in potential is applied there is an inter-atomic exchange of corpuscies and the current flows.

We must look upon a conducting wire as the path along which the free ends of lines of force can move.

The effect of connecting a source of electricity with the ends of the wire is to cause the lines of force to concentrate within the wire thus relieving the stress in the ether.

It is only the negative ends of the lines of force, attached to the corpuscles, that move, the positive ends remaining at rest.

MATTER AND ELECTRICITY.

It was to the genius of Maxwell supplemented by the experimental skill of Hertz that we owe our present knowledge of the intimate relation between light and electricity.

The electro-magnetic theory of light forces us to the consideration of vibrating systems, the frequencies of which are so great that we cannot conceive of the undulations as originating in the vibration of entire atoms.

The complexity of the light emitted by many incandescent elementary substances shows us that the atom must be very complex also. These facts have puzzled many physicists and of the explanations put forward that of Lorentz and Larmor has received greatest attention. They consider the atom as composed of electric units, called electrons, these units moving in closed orbits about a central nucleus. In terms of this theory, light waves originate from an acceleration in the motion of the intra-atomic electrons and as the electrons are isolated electric units it follows that a magnetic field should deflect them in their orbits and thus change the nature of the light emitted. This consequence of the theory of Lorentz and Larmor has been confirmed experimentally by Zeeman, who discovered that the 
magnetic field is capable of doubling and tripling spectral lines.

After J. J. Thomson proved the existence of corpuscles they were at once identified with the electrons of Lorentz and Lar. mor. If we imagine the atom to consist of a congeries of electrons in rapid orbital motion about a central nucleus we are justified in inferring that the stability of such a system is by no means permanent. The acceleration or retardation of the orbital velocities would tend to disturb the stability of the system and disintegration would result.

This conclusion from the theory would lead us to look for a gradual transmutation of the elements.

From the days of the alchemists to the birth of scientific Chemistry the dream of transmuting the baser metals into silver and gold possessed the minds of many experimenters.

Since the time of Lavoisier students of physical science have looked upon the quest as futile and contrary to well established facts. The recent study of radioactive substances, however, has proven beyond question that atomic disintegration is by no means an impossibility. Uranium has come to be looked upon as a patriarch among the elements and its grandchild radium is the father of a family which would confuse any but the specialist to enumerate.

Here again we witness a beautiful experimental confirmation of a mathematical deduction.

Thomson recently has shown that if we imagine the atom to consist of a number of negatively charged particles or electrons placed within a sphere of positive electrification, many of the periodic relations of the elements which were pointed out by Mendelejeff may be deducted. Under the attractive influence of the central positive nucleus and the mutual repulsions of the electrons, different configurations are produced in much the same manner as Mayer's floating magnets can be made to arrange themselves in different patterns according to the number of magnets involved. It is observed that as the number of magnets is increased, patterns of similar form recur and in much the same way Thomson explains the periodicity of properties in Mendelejeff's table.

The recent work of Thomson and Kaufmann gives conclusive evidence that the mass of a body is to be considered as electric inertia or that the most fundamental property of matter becomes a phenomenon of the ether.

These are a few of the more important advances that have been made in physical science within recent times. 